\title{
Simulation of inelastic electron tunneling spectroscopy of single molecules with functionalized tips
}

\author{
A. Garcia-Lekue, ${ }^{1}$ D. Sanchez-Portal, ${ }^{1,2}$ A. Arnau, ${ }^{1,2,3}$ and T. Frederiksen ${ }^{1}$ \\ ${ }^{1}$ Donostia International Physics Center (DIPC), Paseo Manuel de Lardizabal 4, E-20018 San Sebastian, Spain \\ ${ }^{2}$ Centro de Física de Materiales CFM-MPC, Centro Mixto CSIC-UPVIEHU, Paseo Manuel de Lardizabal 5, E-20018 San Sebastian, Spain \\ ${ }^{3}$ Departamento de Física de Materiales UPVIEHU, Facultad de Química, Apdo. 1072, E-20018 San Sebastian, Spain
}

(Received 4 December 2010; published 8 April 2011)

\begin{abstract}
The role of the tip in inelastic electron tunneling spectroscopy (IETS) performed with scanning tunneling microscopes (STM) is theoretically addressed via first-principles simulations of vibrational spectra of single carbon monoxide (CO) molecules adsorbed on $\mathrm{Cu}(111)$. We show how chemically functionalized STM tips modify the IETS intensity corresponding to adsorbate modes on the sample side. The underlying propensity rules are explained using symmetry considerations for both the vibrational modes and the molecular orbitals of the tip and sample. This suggests that single-molecule IETS can be optimized by selecting the appropriate tip orbital symmetry.
\end{abstract}

DOI: 10.1103/PhysRevB.83.155417

PACS number(s): $72.10 .-\mathrm{d}$

\section{INTRODUCTION}

Vibrational spectroscopy with the scanning tunneling microscope (STM) is a powerful technique to study molecules adsorbed on surfaces. ${ }^{1}$ The method combines the high spatial resolution of the STM with the chemical sensitivity of inelastic electron tunneling spectroscopy (IETS) as first reported for molecules incorporated in metal-oxide-metal tunneling junctions. ${ }^{2}$ At low temperatures, molecular vibrations are almost completely frozen but can be excited by tunneling electrons with sufficient energy. By recording the changes in tunneling conductance as a function of applied bias voltage the onsets of distinct phonon emission processes can be detected. In this way spectra recorded on single molecules are vibrational fingerprints that reveal information about chemical species and local environment. Inelastic tunneling electrons from the STM can also be used to induce and control molecular motion $^{3-6}$ and single-molecule chemistry. ${ }^{7}$

Several theoretical methods have been developed to describe IETS-STM, ${ }^{8-13}$ including approaches based on ab initio calculations for many-electron systems. ${ }^{14-17}$ Good quantitative agreement between simulated and experimental data have been reported for many systems, ${ }^{18-21}$ but the spectra are difficult to rationalize due to the complexity of the underlying symmetries of electronic structure and vibrational modes. Therefore, to identify the active vibrational modes in IETS and to understand the corresponding magnitude and sign of the conductance changes various theories of inelastic propensity rules have been developed. ${ }^{21-24}$ The many-body extension of the Tersoff-Hamann approach developed by Lorente and Persson ${ }^{15}$ permits the derivation of selection rules based on the symmetry of the vibrational modes and the molecular orbitals close to the Fermi level. ${ }^{21}$ However, the morphology and chemical composition of the STM tip is often largely unknown in the experiments and, therefore, tip effects can be important for the interpretation of experimental data. ${ }^{25-27}$ For example, chemically functionalized tips were shown to reveal vibrational modes which are not observed with a bare metallic tip. ${ }^{28}$ Consequently, understanding and disentangling the role of the STM tip in IETS measurement are of relevance.

In this paper we investigate theoretically the interplay between symmetries imposed by the electronic structure of the STM tip and the IETS spectra originating from a vibrating molecule adsorbed on the metal sample. Specifically, we consider the prototype system of a CO molecule adsorbed on $\mathrm{Cu}(111)$ in combination with bare and chemically functionalized STM tips. This choice is motivated by the fact that this system has been the subject of research with a variety of surface analysis techniques for decades and that the observed IETS with metallic tips is now well understood. ${ }^{18,29,30}$ We show that chemically functionalized STM tips can increase the resolving power of IETS and suggest the importance of the symmetries imposed by the electronic structure of the tip probe.

The outline of the paper is as follows. In Sec. II we describe the methodology used in the present study to simulate IETS. In Sec. III we report our calculations for several specific junctions. In a first set of calculations (Sec. III A) we examine aligned junctions in which the sample $\mathrm{CO}$ and the molecule on the tip side share a common rotation axis. These results are analyzed in terms of the symmetry and spatial distribution of the most transmitting eigenchannels of the junction to establish the inelastic propensity rules. Then, in Sec. III B , we consider junctions in which the tip has been laterally displaced. This relaxes the symmetry constraints and, therefore, increases the number of allowed transitions contributing to the IETS signal. We find that the symmetry of the scattering states can still be used to understand the IETS signal. However, rather than the global symmetry of these, it is the local symmetry of the tip scattering states in the region where the deformation potential is significant that determines whether or not they are involved in the inelastic scattering. Finally, in Sec. IV we summarize our study and formulate some general predictions for the influence of the tip symmetry in IETS.

\section{THEORY}

We use density functional theory (DFT) combined with nonequilibrium Green's function (NEGF) methods to perform IETS-STM simulations. The generic systems considered for the simulations are shown in Fig. 1. We use a supercell description of the scattering region with a $3 \times 3$ representation 


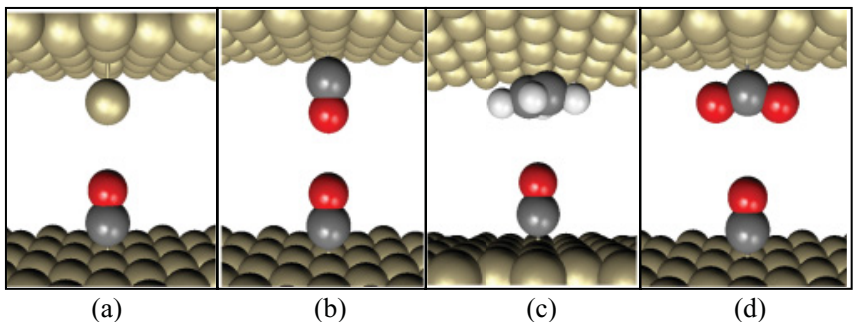

(a) (c)

FIG. 1. (Color online) STM setup: $\mathrm{CO}$ molecule on $\mathrm{Cu}(111)$ with (a) a $\mathrm{Cu}$ adatom tip, or with a tip functionalized with (b) $\mathrm{CO}$, (c) $\mathrm{C}_{2} \mathrm{H}_{4}$, or (d) $\mathrm{CO}_{2}$. Throughout this paper the lower surface with the $\mathrm{CO}$ molecule is considered the STM sample side and the upper surface the tip side.

of a slab containing $6 \mathrm{Cu}(111)$ layers. To represent the STM sample side, we place a single $\mathrm{CO}$ molecule adsorbed on a top site of one of the $\mathrm{Cu}(111)$ surfaces. On the opposite surface, we place a single $\mathrm{Cu}$ adatom [Fig. 1(a)], a carbon monoxide [CO, Fig. 1(b)], an ethylene $\left[\mathrm{C}_{2} \mathrm{H}_{4}\right.$, Fig. 1(c)], or a carbon dioxide $\left[\mathrm{CO}_{2}\right.$, Fig. $\left.1(\mathrm{~d})\right]$ molecule to model the STM tips.

The electronic structure and geometries are calculated with the SIESTA code. ${ }^{31}$ Atomic coordinates of the CO molecule, tip, and surface copper atoms were relaxed until forces were smaller than $0.02 \mathrm{eV} / \AA$. The basis set consisted of single-zeta plus polarization orbitals for $\mathrm{Cu}$ atoms and splitvalence double-zeta plus polarization orbitals for $\mathrm{C}, \mathrm{O}$, and $\mathrm{H}$ atoms. We used the generalized gradient approximation for exchange-correlation, ${ }^{32}$ a cutoff of 200 Ry for the real-space grid integrations, and the $\Gamma$-point approximation for sampling of the three-dimensional Brillouin zone.

The transport properties are simulated with the TRANSIESTA setup, ${ }^{33}$ in which the scattering region described above is coupled to two semi-infinite electrodes on the sample $(S)$ and tip $(T)$ sides, respectively. Since we are only interested in the low-bias regime it suffices to calculate the electronic structure in equilibrium. To keep the present analysis of the inelastic scattering rates transparent we also restrict the transport calculations to the $\Gamma$ point of the two-dimensional Brillouin zone. Eigenchannel visualizations are calculated according to the method described in Ref. 34.

To simulate the inelastic corrections to the tunneling current we employ the INELASTICA package. ${ }^{35,36}$ The vibrational modes $\lambda$ and energies $\hbar \omega_{\lambda}$ are obtained by diagonalizing the dynamical matrix extracted from finite differences for atoms in a predefined vibrational region. Here, we consider two cases: (i) a realistic situation where the sample $\mathrm{CO}$ molecule, the tip structure, and the $\mathrm{Cu}$ atoms in the topmost layers of $\mathrm{Cu}(111)$ surfaces are considered as vibrationally active components of the system, or (ii) an imaginative but illustrative situation where only the sample side ( $\mathrm{CO}$ and topmost $\mathrm{Cu}$ layer) vibrates and the tip side atoms are frozen. For each vibrational mode we determine the corresponding electron-phonon ( $e$-ph) coupling matrix $\mathbf{M}^{\lambda}$ via finite differences of self-consistent Kohn-Sham Hamiltonians. ${ }^{36}$

The inelastic scattering is addressed using the NEGF formalism within the lowest-order-expansion (LOE) scheme, ${ }^{37-39}$ which relies on weak $e$-ph interactions and the electronic

structure at the Fermi level only. Ignoring quasi-elastic correction terms ${ }^{38,39}$ we write the inelastic current as

$$
\begin{aligned}
I^{\mathrm{LOE}}= & G_{0} V \tau \\
& +\sum_{\lambda} I_{\lambda}^{\mathrm{sym}}\left(V, k T,\left\langle n_{\lambda}\right\rangle\right) \operatorname{Tr}\left[\mathbf { G } ^ { \dagger } \boldsymbol { \Gamma } _ { S } \mathbf { G } \left\{\mathbf{M}^{\lambda} \mathbf{A}_{T} \mathbf{M}^{\lambda}\right.\right. \\
& \left.\left.+\frac{i}{2}\left(\boldsymbol{\Gamma}_{T} \mathbf{G}^{\dagger} \mathbf{M}^{\lambda} \mathbf{A} \mathbf{M}^{\lambda}-\text { H.c. }\right)\right\}\right] \\
& +\sum_{\lambda} I_{\lambda}^{\mathrm{asym}}(V, k T) \operatorname{Tr}\left[\mathbf{G}^{\dagger} \boldsymbol{\Gamma}_{S} \mathbf{G}\right. \\
& \left.\times\left\{\boldsymbol{\Gamma}_{T} \mathbf{G}^{\dagger} \mathbf{M}^{\lambda}\left(\mathbf{A}_{T}-\mathbf{A}_{S}\right) \mathbf{M}^{\lambda}+\text { H.c. }\right\}\right] .
\end{aligned}
$$

The first term in Eq. (1) is the elastic Landauer result with transmission $\tau$ times the conductance quantum $G_{0}=2 e^{2} / h$. The second and third terms contain the inelastic corrections to the current from each vibrational mode at the corresponding threshold voltages. The functions $I_{\lambda}^{\mathrm{sym}}\left(V, k T,\left\langle n_{\lambda}\right\rangle\right)$ and $I_{\lambda}^{\text {asym }}(V, k T)$ give rise to symmetric and asymmetric features in the differential conductance $d I / d V$ and depend on bias voltage $V$, fundamental temperature $k T$, and thermal expectation value of the mode occupation $\left\langle n_{\lambda}\right\rangle$. The retarded Green's function $\mathbf{G}$, the spectral function $\mathbf{A}=i\left(\mathbf{G}-\mathbf{G}^{\dagger}\right)$, as well as the electrode couplings $\boldsymbol{\Gamma}_{S, T}$ are all evaluated at the Fermi energy. The quantities $\mathbf{A}_{S, T}=\mathbf{G} \boldsymbol{\Gamma}_{S, T} \mathbf{G}^{\dagger}$ are partial spectral functions defined such that $\mathbf{A}=\mathbf{A}_{S}+\mathbf{A}_{T}$. The sum in Eq. (1) runs over all modes $\lambda$ in the vibrational region.

From the inelastic current $I(V)$ the IETS, defined as

$$
\mathrm{IETS} \equiv \frac{d^{2} I / d V^{2}}{d I / d V},
$$

can be calculated. The normalization by $d I / d V$ is convenient to eliminate the exponential dependence on the tip-substrate distance of the tunneling current. In this way the normalized IETS is a measure of the inelastic cross sections of the various modes. Examination of the "asymmetric" term in Eq. (1) shows that it is zero for symmetric systems. ${ }^{37}$ Experimental IETS curves do often contain asymmetric signals, although the size of these signals is usually small. Such inelastic features can be removed by averaging the IETS signals for positive and negative bias. This symmetrization procedure has been applied to all the IETS spectra reported in Sec. III. Furthermore, our spectra are broadened according to a lock-in signal $V_{\mathrm{rms}}=1.8 \mathrm{mV}$ and a temperature of $T=6 \mathrm{~K}$ (Ref. 36). We note that while the bias inversion symmetry is only approximate for asymmetric junctions, this symmetry is unrelated to the orbital and vibrational symmetries discussed below for the IETS propensity rules.

Our results are useful to understand the effect of the symmetry on the changes in the strength of the IETS signals. With the aim of elucidating the origin of these changes, we have thoroughly investigated the role of the tip electronic structure on the inelastic scattering associated with the vibrational modes of the $\mathrm{CO}$ molecule on the sample side. Nevertheless, caution should be exercised in the quantitative comparison of our simulated IETS spectra with experiments since various approximations are involved in our calculations, such as $\Gamma$-point sampling and LOE approach. Although for most cases our approximations are reasonable, in some situations they might affect position and amplitude of the IETS peaks. 


\section{RESULTS}

In our calculations we have considered the different junctions shown in Fig. 1. The sample is always a flat $\mathrm{Cu}(111)$ surface with an adsorbed $\mathrm{CO}$ molecule. The molecule is assumed to adsorb vertically on a top site of $\mathrm{Cu}(111)$, as experimentally observed. Although such assumption overcomes some of the limitations of many DFT functionals which incorrectly give the fcc hollow as the most stable configuration, ${ }^{40,41}$ it introduces inaccuracies in the prediction of the low-energy frustrated translation (FT) mode. ${ }^{18}$ Therefore, in the following we will focus on the frustrated rotation (FR) and other higherlying vibrational modes, well defined in our DFT-NEGF calculations. ${ }^{18}$

Three conductance channels dominate the elastic transport through the $\mathrm{CO}$ molecule with different rotational symmetry around the molecular axis. As shown in Fig. 2, two channels have $\pi$ character and one has $\sigma$ character. ${ }^{34}$ Since electron propagation can occur through each channel in either direction (from sample to tip or vice versa) there are thus two different current-carrying scattering states or wave functions (denoted $s_{i}$ and $t_{i}$ ) associated with the $i$ th channel. In the STM setup these scattering states are exponentially damped across the tunneling barrier and retain only a tiny amplitude on the transmitted side (related to the transmission coefficient). Among the three dominant sample states $\left\{s_{1}^{\pi}, s_{2}^{\pi}, s_{3}^{\sigma}\right\}$ (i.e., $\mathrm{CO}$ side of the junction) we see in Fig. 2 that the weight of the $s^{\sigma}$ state is strongly reduced in the molecular apex (O atom) compared with the more extended $s^{\pi}$ states. As expected, the symmetry and spatial distribution of the sample states reflect to some extent those of the frontier orbitals of CO: the two-fold degenerate $\pi$-type lowest unoccupied molecular orbital (LUMO), and the $\sigma$-type highest occupied molecular orbital (HOMO) which is more localized on the $\mathrm{C}$ atom.

The symmetry of the channels is an important ingredient to determine the inelastic cross-section associated with a given vibrational mode. ${ }^{24}$ Given the symmetry of the FR mode [ $\pi$ character with respect to the $\mathrm{CO}$ molecule symmetry axis, cf. Fig. 2(e)] we can formulate the selection rules in a quite concise form: sample states with $\pi$ character couple only to tip states with $\sigma$ character around the axis of the sample $\mathrm{CO}$ molecule (i.e., $s_{i}^{\pi} \leftrightarrow t_{j}^{\sigma}$ ) and, conversely, sample states with $\sigma$ character couple only to tip states with $\pi$ character (i.e., $s_{i}^{\sigma} \leftrightarrow t_{j}^{\pi}$ ). ${ }^{42}$ Note that here we always define the $\pi$ or $\sigma$ symmetry character of the transmission channels with respect to the axis normal to the surface (i.e., with respect to the molecular axis) and not in the conventional way for molecular orbitals. Therefore, its meaning might be different, in particular, when the two symmetry axes do not coincide.

It is important to note that symmetry considerations only allow to determine which scattering events are possible. The relative contribution of each allowed transition to the total inelastic cross-section depends on other factors. In particular, inelastic scattering rates strongly depend on the extension of the wave functions of the scattering states (both from sample and tip) along the molecule and toward the vacuum. Since we focus on molecular vibrations, the cross section of each scattering event is determined by the overlap between these scattering states within the gap region, where the molecule is located and the deformation potential associated with the vibration is stronger. From this simple reasoning, and given their different spatial distribution, we can expect that transitions in which $s^{\sigma}$ of the CO molecule is involved will be less efficient that those involving $s^{\pi}$ channels of the same molecule. As we will see, this is indeed confirmed by our calculations.

In the present theoretical work we analyze the effect of changing the symmetry and extension of the tip states via functionalization of a metallic tip with different molecules (cf. $t_{i}$ states in Fig. 2). Our model tip is composed by a flat $\mathrm{Cu}(111)$ surface with or without a $\mathrm{Cu}$ adatom, or with one of these three different molecules: $\mathrm{CO}, \mathrm{CO}_{2}$, and $\mathrm{C}_{2} \mathrm{H}_{4}$. In a first set of calculations, the tip apex is always aligned with the sample $\mathrm{CO}$ molecule, so both sample and tip share a common symmetry axis. The use of these aligned sample-tip arrangements allows us to investigate the effect of the intrinsic symmetry of the tip states on the inelastic transport properties using selection rules. In a second set of calculations, the tips are laterally displaced. The main effect of this displacement is to relax the symmetry constraints of the system, increasing the number of allowed transitions, and to change the distance between the sample and tip molecules, thus affecting the overlap between scattering states coming from both sides. Although the lateral displacement strongly affects the inelastic transport in some cases, it is important to note that such changes can still be completely rationalized using the same ingredients that allow to understand the more symmetric situations: (i) the local character $(\sigma$ or $\pi$ ) of the most conducting tip states around the sample $\mathrm{CO}$ axis, and (ii) the overlap between sample and tip states with the deformation potential. All the results reported in the following correspond to the tunneling regime, with distances between the most protruding tip and sample atoms $\geqslant 3 \AA$.

\section{A. Aligned tips}

Inelastic spectra for $\mathrm{CO} / \mathrm{Cu}(111)$ using a $\mathrm{Cu}$ adatom tip have recently been experimentally and theoretically characterized. ${ }^{18,24}$ We will use this system as a standard reference in what follows. The generic IETS spectrum is dominated by the FR of the CO molecule at $\sim 35 \mathrm{meV}$ (cf. Fig. 3). At lower energies, not considered here, the FT of $\mathrm{CO}$ gives a weaker signal at $\sim 5 \mathrm{meV}$. Similarly, in our simulations with functionalized tips presented below we also find the spectra dominated by the FR of the $\mathrm{CO}$ molecule at $\sim 35 \mathrm{meV}$ (see Fig. 4) for all the different tip terminations, albeit with varying intensity. However, there are also important differences that deserve a careful analysis.

First, we examine the case of the $\mathrm{CO}$ functionalized tip, where the top-most $\mathrm{Cu}(111)$ layers and both tip and sample $\mathrm{CO}$ molecules are allowed to vibrate. The corresponding theoretical IETS spectrum is shown in Fig. 3 together with the result for the $\mathrm{Cu}$ adatom tip. In addition to the FR signal, we find a weaker feature that originates in the center-of-mass (CM) motion ( $\mathrm{Cu}-\mathrm{CO}$ stretch) at $\sim 57 \mathrm{meV}$ (see inset to Fig. 3). Clearly, the use of a $\mathrm{CO}$ functionalized tip enhances the CM signal, which was hardly observed with a $\mathrm{Cu}$ adatom tip. ${ }^{18}$ This effect follows from the selection rules described in Ref. 24, and the observation of such an enhancement in the 

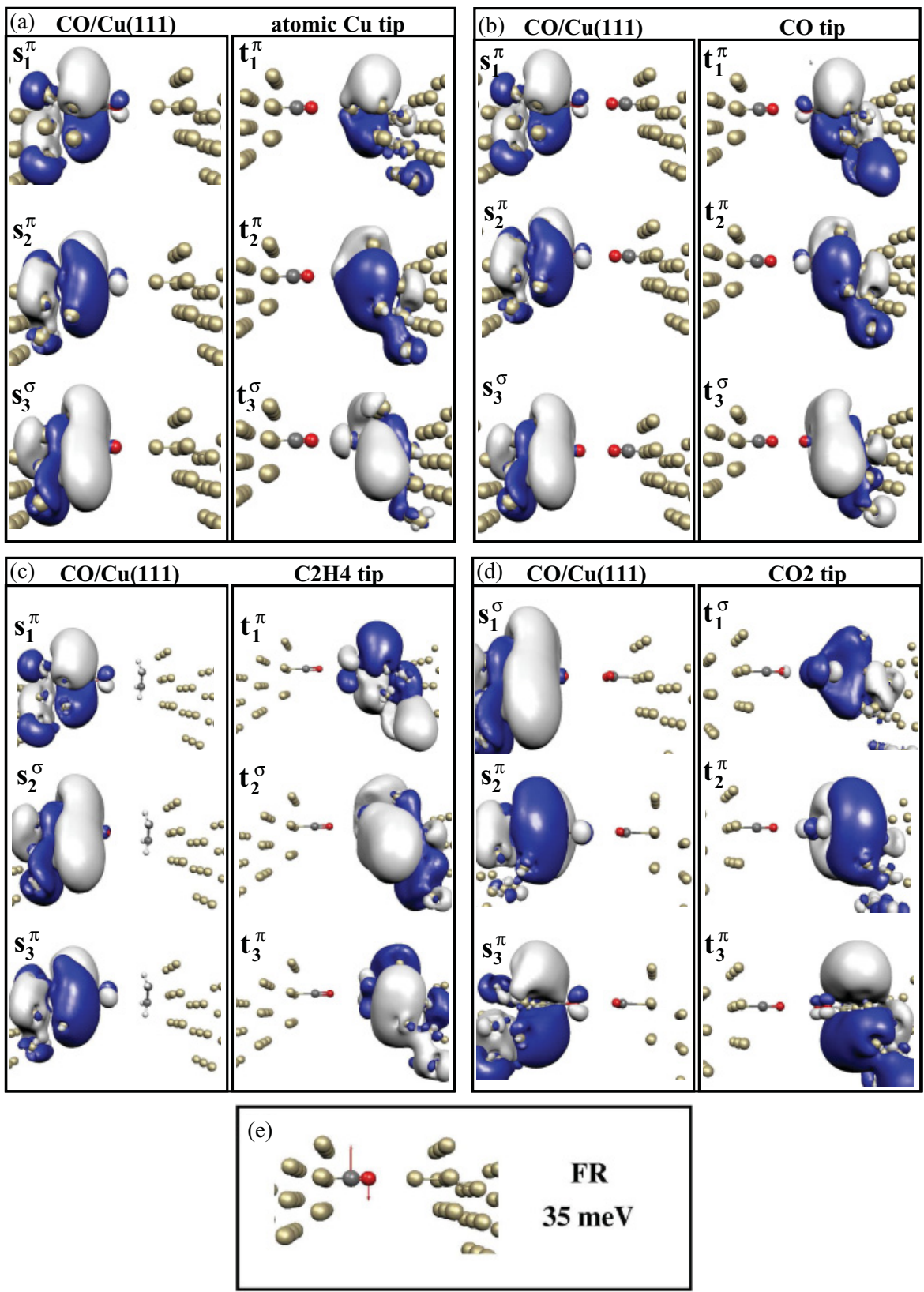

CM signal might be employed for the characterization of the STM tip used in experiments.

To check if the presence of the two $\mathrm{CO}$ molecules at the relatively short tunneling distance used here introduces some coupling of the vibrations or nonlinear effect in the electron-phonon coupling, we have performed a calculation in which one side of the junction is kept frozen. This asymmetric vibrational region yields asymmetric features in the IETS spectrum which we here average out as described above. As seen in Fig. 3, the FR signal obtained when both tip and sample CO molecules vibrate (black line) is larger than the signal obtained with a frozen CO tip (blue dashed curve) by a factor of $\sim 2$, showing that the coupling between the vibrational modes of the two molecules, and its effect in the $e$-ph interaction, is negligible at the considered distances. Therefore, the deformation potential associated with the $\mathrm{CO}$ FR mode is not substantially affected by the presence of neighboring vibrating molecules, and the changes in the calculated IETS spectra using different functionalized tips are mainly related to the different symmetry and spatial distribution of the electronic states in each tip.

Using a CO functionalized tip and a $\operatorname{Ag}(110)$ substrate, $\mathrm{Hahn}$ and $\mathrm{Ho}^{28}$ measured the relative intensity of the FR signal for a CO-vacuum-CO tunnel junction and for a CO-vacuum$\operatorname{Ag}(110)$ tunnel junction. The inelastic signal was observed to be considerably larger in the former case. To check if we can reproduce this behavior, we have also calculated the IETS spectrum using a flat $\mathrm{Cu}$ tip represented by a $\mathrm{Cu}(111)$ surface. Figure 3 shows, in agreement with the experiment, that the corresponding FR signal (red dash-dotted curve) is smaller than the signal obtained with a CO tip (black line). We note that the calculated signal at low energies (around $\pm 20 \mathrm{mV}$ ) originates from the vibrational modes of the $\mathrm{Cu}$ surface and its analysis is out of the scope of this work. ${ }^{43}$ 


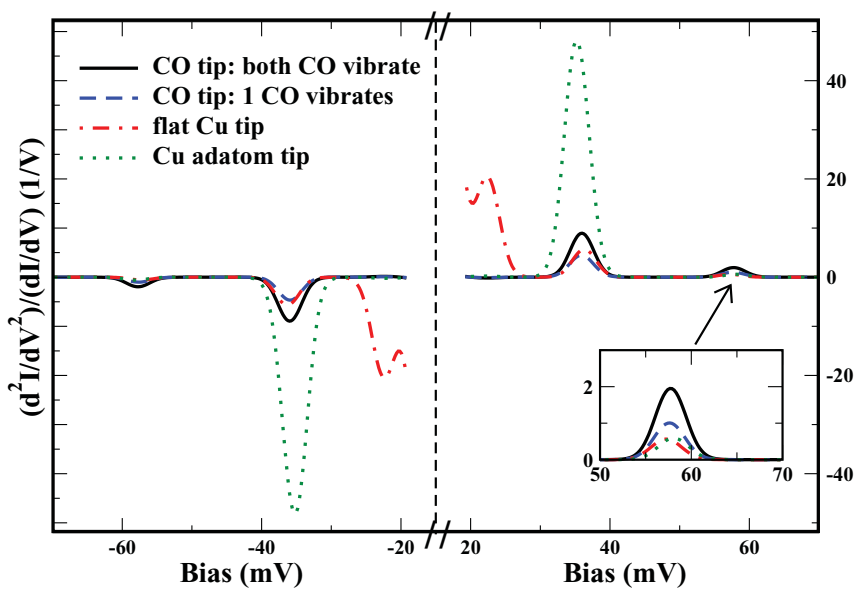

FIG. 3. (Color online) Symmetrized IETS spectra of a CO molecule adsorbed on a $\mathrm{Cu}(111)$ surface with different models for the tip: (i) a CO functionalized tip that is either vibrating (solid line) or frozen (dashed line), (ii) a vibrating flat $\mathrm{Cu}(111)$ tip (dashed-dotted line), or (iii) a vibrating sharp $\mathrm{Cu}$ adatom tip (dotted line). As a reference, the calculated relative change in tunneling conductance due to the frustrated rotation (FR) modes is $24 \%$ with the $\mathrm{Cu}$ adatom tip but only $3 \%$ with the flat $\mathrm{Cu}$ tip. The inset shows a zoom on the signal related to the $\mathrm{CO}$ center-of-mass motion around $57 \mathrm{mV}$.

Next we consider the two other tip terminations shown in Fig. 1. As opposed to the $\mathrm{CO}$ molecule which is oriented perpendicular to the $\mathrm{Cu}(111)$ surface, the $\mathrm{C}_{2} \mathrm{H}_{4}$ and $\mathrm{CO}_{2}$ molecules form almost planar configurations on $\mathrm{Cu}(111) .{ }^{44-46}$ The corresponding IETS spectra are represented in Fig. 4, where the positive voltage region $(V>0)$ corresponds to a realistic vibrational region which includes the sample $\mathrm{CO}$, the tip and the top-most $\mathrm{Cu}$ layers, while the negative voltage region $(V<0)$ shows the results obtained for the imaginative but illustrative case when only the sample side (CO molecule and topmost $\mathrm{Cu}$ layer) vibrates. A comparison of the signals shown for these two types of vibrational regions allows us to conclude that the IETS signal around $\pm 35 \mathrm{mV}$ originates from the FR modes of $\mathrm{CO}$ for all four tip models (the signal amplitude is practically unchanged by freezing the tip atoms). Furthermore, with the $\mathrm{CO}_{2}$ tip frozen the $\mathrm{CO}$ $\mathrm{CM}$ signal shown around $-60 \mathrm{mV}$ is suppressed (see inset to Fig. 4). The larger signal observed with the $\mathrm{CO}_{2}$ tip vibrating (shown for $V>0$ ) thus originates in tip vibrations. The FR signal is stronger for the $\mathrm{CO}_{2}$ tip than for the $\mathrm{Cu}$ adatom tip, which, in turn, is much larger than the signal with $\mathrm{CO}$ or $\mathrm{C}_{2} \mathrm{H}_{4}$ tips. A small FR signal with the $\mathrm{C}_{2} \mathrm{H}_{4}$ tip is consistent with the experimental observations reported in Ref. 28.

Now we discuss our findings using the above-mentioned symmetry considerations for both the vibrational mode, more precisely the corresponding deformation potential, and wave functions of the relevant (tip and sample) scattering states, as well as their spatial extension. The inelastic scattering rate for a given phonon mode $\lambda$ can be expressed as ${ }^{24}$

$$
\gamma_{\lambda}=\frac{4 \pi e}{\hbar} \sum_{i, j}\left|\left\langle s_{i}\left|M^{\lambda}\right| t_{j}\right\rangle\right|^{2}, \quad|e V|>\hbar \omega_{\lambda},
$$

where $M^{\lambda}$ represents the $e$-ph coupling and the summation runs over all sample $\left(s_{i}\right)$ and tip $\left(t_{i}\right)$ scattering states. As

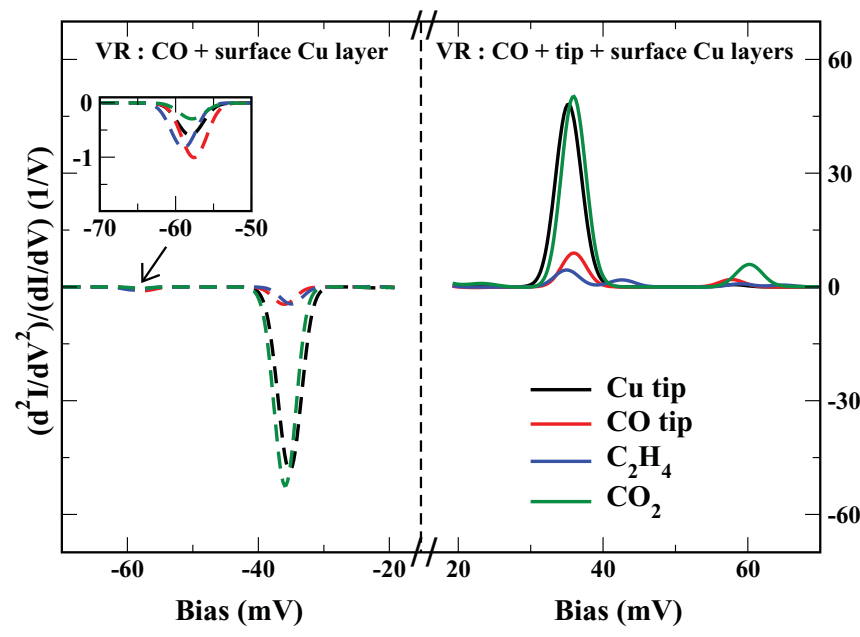

FIG. 4. (Color online) Symmetrized IETS spectra of a CO molecule adsorbed on $\mathrm{Cu}(111)$ surface using a $\mathrm{Cu}$ adatom tip or a tip functionalized with $\mathrm{CO}, \mathrm{C}_{2} \mathrm{H}_{4}$, or $\mathrm{CO}_{2}$. Two vibrational regions (VR) are considered: (i) top-most $\mathrm{Cu}$ layers, sample $\mathrm{CO}$ molecule and tip molecule vibrating (shown for $V>0$ ), and (ii) only sample side vibrating (shown for $V<0$ ). As a reference, the calculated relative change in tunneling conductance due to the frustrated rotation (FR) modes is $25 \%$ with the $\mathrm{CO}_{2}$ tip but only $2 \%$ with the $\mathrm{C}_{2} \mathrm{H}_{4}$ tip. The inset shows a zoom on the signal related to the $\mathrm{CO}$ center-of-mass motion around $-57 \mathrm{mV}$.

reported in Ref. 24, in this eigenchannel basis it suffices to include only the most transmitting eigenchannels for the evaluation of Eq. (3). This is due to the fact that only these scattering states have significant spatial weight in the molecular part of the junction where the $e$-ph interaction (i.e., deformation potential) associated with the molecular vibrations is localized. In Fig. 2 we visualize the three most transmitting eigenchannels as represented by scattering states originating from the $\mathrm{CO}$ sample side $\left(s_{i}\right)$ or from the tip side $\left(t_{i}\right)$. By construction the scattering states belonging to a given eigenchannel have the same symmetry. As one can see in Fig. 2, the three dominant channels are either $\sigma$ or $\pi$ type.

For each vibrational mode it follows from Eq. (3) that only scattering states with certain symmetry can couple, giving rise to the so-called selection or propensity rules. ${ }^{24}$ The two-fold degenerate FR mode corresponds to the rotation of the $\mathrm{CO}$ molecule parallel to the $x y$ plane. The associated deformation potential has a $\pi$ character with respect to the $\mathrm{CO}$ axis. Correspondingly, the FR mode can only produce scattering of electrons between $\pi$ - and $\sigma$-type channels. This simple picture is confirmed by our numerical calculations for the aligned junctions. Table I gives the calculated contributions of the transitions between the three most transmitting eigenchannels to the total inelastic scattering rate of the two FR modes of $\mathrm{CO}$, as obtained with different tips. For the $\mathrm{Cu}$ adatom tip the main contribution to the inelastic scattering comes from the transitions $\left\{s_{1}^{\pi}, s_{2}^{\pi}\right\} \leftrightarrow t_{3}^{\sigma}$ and amounts to $\sim 95 \%$ of the total scattering rate. Similarly, for the $\mathrm{CO}_{2}$ tip the main contribution is $\left\{s_{2}^{\pi}, s_{3}^{\pi}\right\} \leftrightarrow t_{1}^{\sigma}(\sim 98 \%)$. In these two cases the dominant tip state has $\sigma$ character, while the sample states have $\pi$ character. On the contrary, in the case of the $\mathrm{C}_{2} \mathrm{H}_{4}$ tip the dominant transition is $s_{2}^{\sigma} \leftrightarrow t_{1}^{\pi}(\sim 73 \%)$. For 
TABLE I. Decomposition (in \%) of the total inelastic scattering rate for the frustrated rotations $\left(\mathrm{FR}_{1}\right.$ and $\left.\mathrm{FR}_{2}\right)$ of $\mathrm{CO}$ on $\mathrm{Cu}(111)$ among the three dominant scattering states only (i.e., a $3 \times 3$ matrix). The sum over all nine matrix elements, stated next to the tip label, does not reach $100 \%$ because of the total inelastic scattering rate also involves scattering with the other, less-conducting channels not analyzed in this work. The elastic transmission coefficient for each channel is also given $\left(\tau_{i}=\tau_{i}^{s}=\tau_{i}^{t}\right)$.

\begin{tabular}{lccc}
\hline \hline Cu tip (99.4) & $t_{1}^{\pi}$ & $t_{2}^{\pi}$ & $t_{3}^{\sigma}$ \\
$s_{1}^{\pi}$ & 0.0 & 0.1 & 46.8 \\
$s_{2}^{\pi}$ & 0.0 & 0.1 & 47.8 \\
$s_{3}^{\sigma}$ & 2.2 & 2.3 & 0.1 \\
$\tau_{i} / 10^{-4}$ & 3.74 & 3.73 & 3.33 \\
\hline $\mathbf{C O ~ t i p ~ ( 9 4 . 1 ) ~}$ & $t_{1}^{\pi}$ & $t_{2}^{\pi}$ & $t_{3}^{\sigma}$ \\
$s_{1}^{\pi}$ & 0.0 & 0.0 & 22.7 \\
$s_{2}^{\pi}$ & 0.0 & 0.0 & 22.8 \\
$s_{3}^{\sigma}$ & 22.8 & 22.9 & 0.0 \\
$\tau_{i} / 10^{-4}$ & 1.02 & 1.02 & 0.10 \\
\hline $\mathbf{C}_{2} \mathbf{H}_{4}$ tip (80.8) & $t_{1}^{\pi}$ & $t_{2}^{\sigma}$ & $t_{3}^{\pi}$ \\
$s_{1}^{\pi}$ & 0.0 & 0.4 & 0.1 \\
$s_{2}^{\sigma}$ & 72.5 & 0.0 & 4.8 \\
$s_{3}^{\pi}$ & 0.2 & 2.1 & 0.3 \\
$\tau_{i} / 10^{-4}$ & 8.18 & 0.88 & 0.63 \\
\hline $\mathbf{C O}_{2}$ tip (99.8) & $t_{1}^{\sigma}$ & $t_{2}^{\pi}$ & $t_{3}^{\pi}$ \\
$s_{1}^{\sigma}$ & 0.0 & 1.9 & 0.1 \\
$s_{2}^{\pi}$ & 39.1 & 0.0 & 0.0 \\
$s_{3}^{\pi}$ & 58.6 & 0.0 & 0.0 \\
$\tau_{i} / 10^{-4}$ & 5.68 & 2.53 & 0.20 \\
\hline \hline
\end{tabular}

the CO tip, since sample and tip subsystems are identical, the dominant contribution $(\sim 94 \%)$ is given by the sum of transitions between $\left\{s_{1}^{\pi}, s_{2}^{\pi}\right\} \leftrightarrow t_{3}^{\sigma}$ and between $s_{3}^{\sigma} \leftrightarrow\left\{t_{1}^{\pi}, t_{2}^{\pi}\right\}$.

In the case of the $\mathrm{Cu}$ adatom and $\mathrm{CO}$ tips [see Figs. 1(a) and 1(b)] there exists an axial symmetry in the junction due to their vertical adsorption and the hexagonal symmetry of the substrate. This implies that the two $\pi$ eigenchannels and the two FR modes are identical. For the $\mathrm{Cu}$ adatom tip, the tip state $t_{3}^{\sigma}$ extends more toward vacuum than $t_{1}^{\pi}$ and $t_{2}^{\pi}$ [cf. Fig. 2(a)]. In principle, we can relate the $\sigma$ channel to the $4 s$ orbital of the $\mathrm{Cu}$ adatom, for which we can expect to find a considerable spectral weight at energies nearby the Fermi energy. According to the selection rules, this tip state can couple with the sample states $\left\{s_{1}^{\pi}, s_{2}^{\pi}\right\}$. Since $s_{1}^{\pi}$ and $s_{2}^{\pi}$ also extend the most along the molecule and into vacuum, the transitions $\left\{s_{1}^{\pi}, s_{2}^{\pi}\right\} \leftrightarrow t_{3}^{\sigma}(\sim 95 \%)$ completely dominate the inelastic scattering. As expected by symmetry, each of these two transitions contribute almost equally to the total inelastic rate. For the same reason, the transitions $s_{3}^{\sigma} \leftrightarrow\left\{t_{1}^{\pi}, t_{2}^{\pi}\right\}(\sim 2 \%)$ also contribute equally, although in a much smaller percentage due to the smaller overlap of $s_{3}^{\sigma}$ with the vibrational region and with $\left\{t_{1}^{\pi}, t_{2}^{\pi}\right\}$ of the $\mathrm{Cu}$ adatom tip. With the same axial symmetry, the situation is similar in the case of the CO tip, where the transitions $s_{3}^{\sigma} \leftrightarrow t_{1}^{\pi}, s_{3}^{\sigma} \leftrightarrow t_{2}^{\pi}, s_{1}^{\pi} \leftrightarrow t_{3}^{\sigma}$, and $s_{2}^{\pi} \leftrightarrow t_{3}^{\sigma}$ yield a $\sim 23 \%$ contribution each (i.e., in this case tip and sample states with both symmetries become relevant). However, since with two $\mathrm{CO}$ in the junction the $\sigma$-type states are always rather localized near the metallic surface, the inelastic signal is significantly reduced with respect to that of the $\mathrm{Cu}$ adatom tip. This is so, even if we take into account that, with two vibrating $\mathrm{CO}$ molecules in the junction, the inelastic signal associated with the FR modes of $\mathrm{CO}$ doubles with respect to that of a single vibrating $\mathrm{CO}$ molecule.

Although in this paper we mainly focus on the FR mode of $\mathrm{CO}$, the reason for the intensity increase of the $\mathrm{CM}$ mode when a CO tip is used also deserves some discussion. Contrary to the case of the FR mode, the deformation potential associated with the CM mode has $\sigma$ character around the molecular axis and induces transitions between tip and sample channels of the same symmetry (i.e., $s_{i}^{\sigma} \leftrightarrow t_{j}^{\sigma}$ or $s_{i}^{\pi} \leftrightarrow t_{j}^{\pi}$ ). Using a CO tip, the inelastic scattering induced by the CM mode is dominated by the more extended $\pi$ channels of $\mathrm{CO}$. Using a $\mathrm{Cu}$ adatom tip, the most important transition induced by the $\mathrm{CM}$ mode is $s_{3}^{\sigma} \leftrightarrow t_{3}^{\sigma}$. However, these $\sigma$ channels have a much smaller overlap as compared with the $\pi$ channels that dominate the case of the CO tip. For this reason, the CO tip considerably enhances the $\mathrm{CM}$ signal.

As for $\mathrm{CO}_{2}$ and $\mathrm{C}_{2} \mathrm{H}_{4}$ tips, they exhibit in-plane adsorption on $\mathrm{Cu}(111)$ and hence, define a preferential direction given by the molecular axis within the $x y$ plane. Consequently, the two $\pi$ channels are not identical anymore and the scattering cross section of the two FR modes also differs. In the case of the $\mathrm{CO}_{2}$ tip the most important processes are the transitions $\left\{s_{2}^{\pi}, s_{3}^{\pi}\right\} \leftrightarrow t_{1}^{\sigma}(\sim 98 \%)$ (i.e., between sample $\pi$ states and tip $\sigma$ states, just as in the case of the $\mathrm{Cu}$ adatom tip). However, the contribution from $s_{3}^{\pi} \leftrightarrow t_{1}^{\sigma}$ is larger than the contribution from $s_{2}^{\pi} \leftrightarrow t_{1}^{\sigma}$. This symmetry breaking is also observed for the $s_{1}^{\sigma} \leftrightarrow t_{3}^{\pi}$ component, which is almost negligible in comparison with the $s_{1}^{\sigma} \leftrightarrow t_{2}^{\pi}$ component. In the case of $\mathrm{C}_{2} \mathrm{H}_{4}$, we find that the transition $s_{2}^{\sigma} \leftrightarrow t_{1}^{\pi}$ amounts to $\sim 73 \%$ of the total inelastic rate while $s_{2}^{\sigma} \leftrightarrow t_{3}^{\pi}$ only gives $\sim 5 \%$. This difference is readily understood from Fig. 2(c) where one notes that $t_{1}^{\pi}$ extends more toward the CO sample than the $t_{3}^{\pi}$ state, and hence has the largest overlap with the vibrational region.

All these results provide numerical evidence of the selection rules governing the inelastic scattering processes. Furthermore, as the $e$-ph coupling is local in space, even if the symmetry of the orbitals allows for a transition, the sample and tip states must also extend quite appreciably in the transport direction in order to yield a significant inelastic signal. Therefore, we have seen that it is the combination of the symmetry and spatial extension of the tip and sample states that ultimately determines the IETS intensity. This is indeed what is reported in Fig. 4, where the FR signal is smallest for the $\mathrm{C}_{2} \mathrm{H}_{4}$ tip, due to a strong attenuation of the $t^{\sigma}$ state along the molecular axis as compared to, for example, $t^{\sigma}$ for a $\mathrm{Cu}$ tip. On the contrary, for the $\mathrm{CO}_{2}$ tip the $t_{1}^{\sigma}$ state extends deeper into the vibrational region and thus allows for a stronger inelastic FR signal.

\section{B. Laterally displaced tips}

In the following we consider junctions with laterally displaced tips, shown in the lower panels in Fig. 5. If the tip is slightly displaced from the aligned arrangements considered in Sec. III A [see Fig. 1], the symmetry of the tip-sample system is broken. For the $\mathrm{Cu}$ adatom or $\mathrm{CO}$ tips this means 

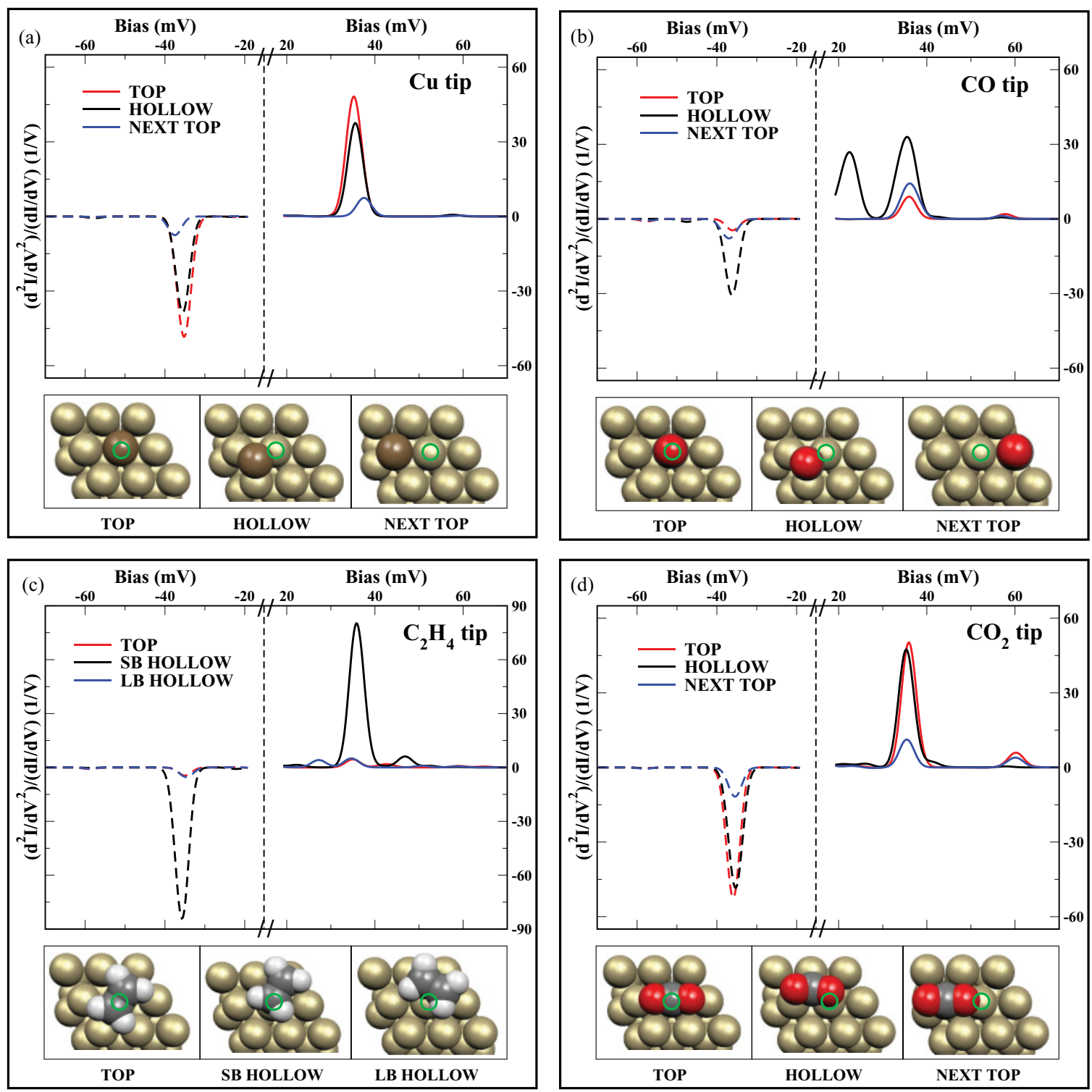

FIG. 5. (Color online) Symmetrized IETS spectra for a $\mathrm{CO}$ molecule adsorbed on $\mathrm{Cu}(111)$ surface using (a) a $\mathrm{Cu}$ adatom tip, (b) a CO, (c) a $\mathrm{C}_{2} \mathrm{H}_{4}$, or (d) a $\mathrm{CO}_{2}$ tip placed on different positions of $\mathrm{Cu}(111)$, as shown at the bottom part of each figure. Two vibrational regions are considered: (i) top-most $\mathrm{Cu}$ layers, $\mathrm{CO}$ molecule and tip molecule (shown for $V>0$ ), and (ii) only sample $\mathrm{CO}$ molecule (shown for $V<0$ ). As a reference, the calculated relative change in tunneling conductance due to the frustrated rotation (FR) modes is $24 \%$ with the $\mathrm{Cu}$ adatom tip (top site). The (green) open circle indicates the position of the sample $\mathrm{CO}$ molecule, which always remains on a $\mathrm{Cu}$ top site on the opposite surface.

that we no longer have a common rotation axis in these junctions. Similarly, for the $\mathrm{CO}_{2}$ and $\mathrm{C}_{2} \mathrm{H}_{4}$ tips (which do not carry rotational symmetry) it means that we reduce the number of symmetry operations. As illustrated in Fig. 5, we are considering the following laterally displaced tip-sample configurations: (i) hollow and next-top configurations for atomic $\mathrm{Cu}, \mathrm{CO}$ and $\mathrm{CO}_{2}$ tips, and (ii) short-bridge and long-bridge hollow configurations for the $\mathrm{C}_{2} \mathrm{H}_{4}$ tip. We note that the $\mathrm{Cu}(111)$ surface and the tip cannot easily be laterally displaced due to the use of periodic boundary conditions in SIESTA. This would require the use of a substantially larger unit cell (e.g., the use of two slabs instead of one, or the inclusion of a dislocation in a sufficiently thick slab, such that the dislocation does not affect the surface electronic structure). The use of such a large supercell would significantly increase the computational cost of the calculations. Instead, we have decided here to use a single metallic slab. This constraint keeps the computational load at a moderate level and facilitates the comparison with calculations of the aligned configuration, although forces us to study only discrete translations of the functional part of the tip from one adsorption site to the nearest one.

The results for the corresponding inelastic spectra are also shown in Fig. 5. One sees that the intensity of the inelastic signal strongly depends on the position of the tip. In some cases, we also see slight variations of the energy of the $\mathrm{CO}$ FR mode. These small variations are consistent with those recently observed in Ref. 18 when the size of the tunneling 
gap is changed. However, in the following we concentrate only in the reasons behind the change of the intensity of the inelastic signal.

In the case of the $\mathrm{Cu}$ adatom and the $\mathrm{CO}_{2}$ tips [see Figs. 5(a) and 5(d)], the corresponding IETS spectra exhibit almost no change when displacing the tip from top to hollow position. As already shown in Sec. III A, the most important tip states for FR scattering with these two types of tips are the $t^{\sigma}$ states with $\sigma$ symmetry. Therefore, as the tip moves away from the top position, the main effect is a modification of the overlap between a $t^{\sigma}$-type state and the deformation potential associated with the FR modes. Such an overlap does not change appreciably as the $\mathrm{Cu}$ adatom or $\mathrm{CO}_{2}$ tips move from top to hollow positions and, as a result, the corresponding FR signal is hardly modified. However, when the tips are further displaced to the next-top position the overlap decreases much more and, consequently, the FR signal is reduced. In principle, due to the reduction of symmetry the $\sigma$ and $\pi$ classification is only approximate and all transitions among the scattering states $\left(s_{i} \leftrightarrow t_{j}\right)$ are allowed. But these contributions are small in practice. Our calculations suggest that when a $t^{\sigma}$ tip state gives the largest contribution to the inelastic signal of the FR mode of $\mathrm{CO}$, this signal will decrease as the tip is laterally displaced following the reduction of the overlap between the electronic states of the tip and sample.

The IETS spectra with $\mathrm{CO}$ and $\mathrm{C}_{2} \mathrm{H}_{4}$ tips present a different behavior as the tip-sample relative positions change [see Figs. 5(b) and 5(c)]. The main difference, as compared to the previously discussed cases of $\mathrm{Cu}$ adatom and $\mathrm{CO}_{2}$ tips, is that, the FR signal increases when the tip is laterally displaced away from the aligned position. The main factor behind this behavior is the increase in the number of allowed transitions when the symmetry is reduced. In the case of $\mathrm{CO}$, as the tip is laterally displaced from its original top position to hollow site and further to next top, the FR signal first increases significantly and then decreases practically to its initial value. The first increase is easy to interpret: when the molecule is displaced inelastic scattering between tip and sample $\pi$-like states becomes allowed $(\pi \leftrightarrow \pi)$. Since the $\pi$ states extend the most into the tunneling gap this transition is more efficient than $\sigma \leftrightarrow \pi$, which were the only allowed transitions for the aligned configuration. Besides, there is a signal at $\sim \pm 20 \mathrm{mV}$ caused by vibrations of the $\mathrm{Cu}$ atoms in the $\mathrm{Cu}(111)$ surface layers of the sample and the tip, similar to the one observed with a flat $\mathrm{Cu}(111)$ tip [see Fig. 3]. By further displacing the tip to the next top position we see a decrease in the FR signal, consistent with a decrease of the overlap between tip states with sample states as well as the vibrational region.

A similar behavior is observed in Fig. 5(c) in the case of $\mathrm{C}_{2} \mathrm{H}_{4}$ functionalization. When the tip molecule is located on top site or long-bridge hollow site the FR signal is almost identical and small, but dramatically enhanced if we move the tip to a short-bridge hollow position. To understand the origin of this strong enhancement of the FR peak we analyze the spatial properties of the dominant tip state $\left(t_{1}\right)$ for each of the three $\mathrm{C}_{2} \mathrm{H}_{4}$ tip positions. Figure 6 shows a top view of the three different tip states, where the origin (green empty circle) corresponds to the position of the sample $\mathrm{CO}$ molecule. This allows us to survey the "local" symmetry character of the tip states with respect to the center of the $\mathrm{CO}$ vibrational mode.

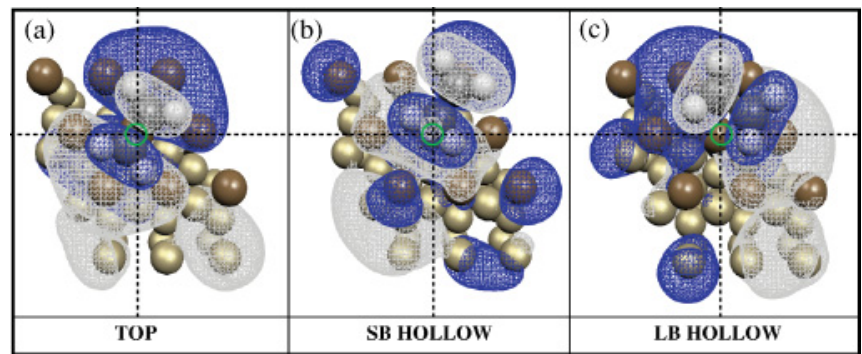

FIG. 6. (Color online) Top view of the tip scattering state which contributes the most to the inelastic scattering rate. The green empty circle represents the $x y$ position of the sample $\mathrm{CO}$ molecule. The panels correspond to the $\mathrm{C}_{2} \mathrm{H}_{4}$ molecule placed on (a) top site, (b) short-bridge hollow, and (c) long-bridge hollow positions [as shown in the lower panel of Fig. 5(c)]. For clarity, the $\mathrm{Cu}$ atoms on the first layer underneath the tip have been represented in dark brown. The blue and white colors represent the signs of the real part of scattering states (due to the choice of phase the imaginary part is negligible for visualization purposes).

As mentioned in the beginning of Sec. III this is the critical feature responsible for the amplitude of the IETS signal of the FR mode. In Figs. 6(a) and 6(c) we observe that for top and long-bridge hollow tip arrangements, the dominant tip state has a local $\pi$-type symmetry. For this reason the FR mode cannot couple it efficiently to the $\pi$-type CO sample states, hence the small FR inelastic signal in Fig. 5(c). On the contrary, when the $\mathrm{C}_{2} \mathrm{H}_{4}$ molecule is placed on the short-bridge hollow position [see Fig. 6(b)] the dominant eigenchannel has a strong local $\sigma$-type character and therefore yields a significantly stronger FR signal. The IETS peak for the FR modes of the CO is even larger than the one observed with a $\mathrm{Cu}$ adatom or a $\mathrm{CO}_{2}$ tip on top positions, due to the fact that the involved tip states extend even further into the vibrational region.

\section{CONCLUSION}

In this work the role of the STM tip on inelastic electron tunneling spectroscopy has been thoroughly investigated. Special emphasis has been placed on the effects of changes in symmetry of the orbitals involved in the inelastic scattering processes.

We have simulated IETS spectra for a CO molecule placed on a $\mathrm{Cu}(111)$ surface using a $\mathrm{Cu}$ adatom tip as well as tips functionalized with different molecules $\left(\mathrm{CO}, \mathrm{CO}_{2}\right.$, and $\left.\mathrm{C}_{2} \mathrm{H}_{4}\right)$. For all the different tips considered here, the IETS spectra are dominated by the excitation of the frustrated rotation vibrational mode of the $\mathrm{CO}$ molecule at $\sim 35 \mathrm{meV}$. However, we find that the intensity of the FR peak strongly depends on the chemical nature as well as on the position of the STM tip. These effects were rationalized by means of the propensity rules, that is, taking into account the local symmetry character of both the deformation potential associated with the excited vibrational mode and of the most transmitting scattering states associated with the tip and sample, in the region where the deformation potential is appreciable.

We performed a detailed analysis of these effects by visualizing the eigenchannels of the different junctions. In most cases the symmetry and the spatial distribution of these dominant scattering states can be rationalized in simple 
terms and correlated with the frontier molecular orbitals. Specifically, our analysis suggests the following consequences of IETS propensity rules related to the symmetry respect to the surface normal: adsorbate molecules for which $\pi$-type frontier orbitals control the electron transport reveal IETS signals from transversal (longitudinal) vibrational modes if observed with tips of $\sigma$-type ( $\pi$-type) states. Conversely, adsorbate molecules with dominating $\sigma$-type frontier orbitals reveal IETS signals from longitudinal modes if observed with $\sigma$ tips and transverse modes if observed with $\pi$ tips. In short, rotational symmetry around the transport direction favors inelastic transitions where the product of sample state, deformation potential, and tip state is symmetric. Note that the $\pi$ or $\sigma$ symmetry character of the transmission channels is defined with respect to the axis normal to the surface and, therefore, its meaning is not always the same as the conventional one of the molecular orbitals.

Our calculations further show that, for a given vibrational mode of the adsorbed molecule, the inelastic scattering rate is mainly determined by the local character in the vibrational region of the tip channels and that, as a result, the relative tip-sample configuration is crucial. Therefore, in the general cases where sample and tip do not share a symmetry axis, we have shown that the propensity rules stated above apply approximately in terms of the "local" symmetry in the region of space where the molecular vibrations occur.

For the particular case of the frustrated rotation of $\mathrm{CO}$, the locality effect is specially relevant if the tip states have dominantly $\pi$-type character because then the inelastic signal can be largely increased by simply shifting the tip laterally. We have also found that, in some cases, functionalized tips can enhance inelastic signals as compared to those obtained with a metallic adatom tip. For instance, the signal from the center-of-mass motion of one $\mathrm{CO}$ molecule was found to be larger when observed with a CO tip than with a metallic tip (cf. inset to Fig. 3).

In conclusion, we have shown that the STM tip is of vital importance in inelastic tunneling spectroscopy and that, as a result, single-molecule vibrational spectroscopy could be modulated and manipulated by means of functionalized STM tips. Moreover, since the effect of the tip might play an important role in the IETS measurements, it would be desirable to take it into account in the interpretation of experimental data. We believe our results can help in that direction.

\section{ACKNOWLEDGMENTS}

We thank N. Lorente, M. Paulsson, and H. Ueba for stimulating discussions. Support from the Basque Departamento de Educación, UPV/EHU (Grant No. IT-366-07), the Spanish Ministerio de Ciencia e Innovación (Grant No. FIS2010-19609-C02-00), the ETORTEK program funded by the Basque Departamento de Industria and the Diputacion Foral de Guipuzcoa are gratefully acknowledged.
${ }^{1}$ B. C. Stipe, R. A. Rezaei, and W. Ho, Science 280, 1732 (1998).

${ }^{2}$ R. C. Jaklevic and J. Lambe, Phys. Rev. Lett. 17, 1139 (1966).

${ }^{3}$ T. Komeda, Y. Kim, M. Kawai, B. N. J. Persson, and H. Ueba, Science 295, 2055 (2002).

${ }^{4}$ A. J. Heinrich, C. P. Lutz, J. A. Gupta, and D. M. Eigler, Science 298, 1381 (2002).

${ }^{5}$ J. I. Pascual, N. Lorente, Z. Song, H. Conrad, and H. P. Rust, Nature (London) 423, 525 (2003).

${ }^{6}$ N. Lorente, R. Rurali, and H. Tang, J. Phys.: Condens. Matt. 17, S1049 (2005).

${ }^{7}$ W. Ho, J. Chem. Phys. 117, 11033 (2002).

${ }^{8}$ J. Appelbaum and W. Brinkman, Phys. Rev. 186, 464 (1969).

${ }^{9}$ C. Caroli, R. Combescot, P. Nozières, and D. Saint-James, J. Phys. C 5, 21 (1972).

${ }^{10}$ B. N. J. Persson and A. Baratoff, Phys. Rev. Lett. 59, 339 (1987).

${ }^{11}$ J. Bŏnca and S. A. Trugman, Phys. Rev. Lett. 75, 2566 (1995).

${ }^{12}$ E. G. Emberly and G. Kirczenow, Phys. Rev. B 61, 5740 (2000).

${ }^{13}$ M. Galperin, M. A. Ratner, and A. Nitzan, J. Chem. Phys. 121, 11965 (2004).

${ }^{14}$ N. Mingo and K. Makoshi, Phys. Rev. Lett. 84, 3694 (2000).

${ }^{15}$ N. Lorente and M. Persson, Phys. Rev. Lett. 85, 2997 (2000).

${ }^{16}$ T. Frederiksen, M. Brandbyge, N. Lorente, and A.-P. Jauho, Phys. Rev. Lett. 93, 256601 (2004).

${ }^{17}$ M. Alducin, D. Sánchez-Portal, A. Arnau, and N. Lorente, Phys. Rev. Lett. 104, 136101 (2010).

${ }^{18}$ L. Vitali, R. Ohmann, K. Kern, A. Garcia-Lekue, T. Frederiksen, D. Sanchez-Portal, and A. Arnau, Nano Lett. 10, 657 (2010).

${ }^{19}$ N. Okabayashi, M. Paulsson, H. Ueba, Y. Konda, and T. Komeda, Phys. Rev. Lett. 104, 077801 (2010).
${ }^{20}$ C. R. Arroyo, T. Frederiksen, G. Rubio-Bollinger, M. Vélez, A. Arnau, D. Sánchez-Portal, and N. Agraït, Phys. Rev. B 81, 075405 (2010).

${ }^{21}$ N. Lorente, M. Persson, L. J. Lauhon, and W. Ho, Phys. Rev. Lett. 86, 2593 (2001).

${ }^{22}$ A. Troisi and M. A. Ratner, Nano Lett. 6, 1784 (2006).

${ }^{23}$ A. Gagliardi, G. C. Solomon, A. Pecchia, T. Frauenheim, A. Di Carlo, N. S. Hush, and J. R. Reimers, Phys. Rev. B 75, 174306 (2007).

${ }^{24}$ M. Paulsson, T. Frederiksen, H. Ueba, N. Lorente, and M. Brandbyge, Phys. Rev. Lett. 100, 226604 (2008).

${ }^{25}$ L. Bartels, G. Meyer, and K. H. Rieder, Surf. Sci. 432, L621 (1999).

${ }^{26}$ G. Teobaldi, M. Peñalba, A. Arnau, N. Lorente, and W. A. Hofer, Phys. Rev. B 76, 235407 (2007).

${ }^{27}$ F. Calleja, A. Arnau, J. J. Hinarejos, A. L. Vazquez de Parga, W. A. Hofer, P. M. Echenique, and R. Miranda, Phys. Rev. Lett. 92, 206101 (2004)

${ }^{28}$ J. R. Hahn and W. Ho, Phys. Rev. Lett. 87, 196102 (2001).

${ }^{29}$ L. J. Lauhon and W. Ho, Phys. Rev. B 60, R8525 (1999).

${ }^{30}$ M. Persson, Phil. Trans. R. Soc. Lond. A. 362, 1173 (2004).

${ }^{31}$ J. M. Soler, E. Artacho, J. D. Gale, A. Garcia, J. Junquera, P. Ordejón, and D. Sanchez-Portal, J. Phys.: Condens. Matter 14, 2745 (2002).

${ }^{32}$ J. P. Perdew, K. Burke, and M. Ernzerhof, Phys. Rev. Lett. 77, 3865 (1996).

${ }^{33}$ M. Brandbyge, J. L. Mozos, P. Ordejón, J. Taylor, and K. Stokbro, Phys. Rev. B 65, 165401 (2002). 
${ }^{34}$ M. Paulsson and M. Brandbyge, Phys. Rev. B 76, 115117 (2007).

${ }^{35}$ [https://sourceforge.net/projects/inelastica].

${ }^{36}$ T. Frederiksen, M. Paulsson, M. Brandbyge, and A.-P. Jauho, Phys. Rev. B 75, 205413 (2007).

${ }^{37}$ M. Paulsson, T. Frederiksen, and M. Brandbyge, Phys. Rev. B 72, 201101(R) (2005).

${ }^{38}$ J. K. Viljas, J. C. Cuevas, F. Pauly, and M. Häfner, Phys. Rev. B 72, 245415 (2005).

${ }^{39}$ F. Haupt, T. Novotný, and W. Belzig, Phys. Rev. B 82, 165441 (2010).

${ }^{40}$ N. Lopez and J. K. Nørskov, Surf. Sci. 477, 59 (2001).

${ }^{41}$ M. Alcántara Ortigoza, R. Heid, K.-P. Bohnen, and T. S. Rahman, Phys. Rev. B 79, 125432 (2009).
${ }^{42}$ Note that inelastic scattering among sample or tip states (i.e., $s_{i}$ $\leftrightarrow s_{j}$ or $t_{i} \leftrightarrow t_{j}$ ) does not yield a bias-dependent signal since their occupations are characterized by the same chemical potential. Such processes can thus safely be ignored in the discussion of inelastic selection rules.

${ }^{43}$ L. Vitali, S. D. Borisova, G. G. Rusina, E. V. Chulkov, and K. Kern, Phys. Rev. B 81, 153409 (2010).

${ }^{44}$ M. Witiko and K. Hermann, Appl. Catal A: Gen. 172, 85 (1998).

${ }^{45}$ O. Skibbe, D. Vogel, M. Binder, A. Pucci, T. Kravchuk, L. Vattuone, V. Venugopal, A. Kokalj, and M. Rocca, J. Chem. Phys. 131, 024701 (2009).

${ }^{46}$ G.-C. Wang, L. Jiang, Y. Morikawa, J. Nakamura, Z.-S. Cai, Y.-M. Pan, and X.-Z. Zhao, Surf. Sci. 570, 205 (2004). 